\title{
Magnetic Torque Induced High/Low Accretion States in AM Herculis Binaries
}

\author{
Warren M. Sparks \\ Los Alamos National Lab, Los Alamos, NM 87545, USA \\ Steve B. Howell \\ University of Wyoming, Laramie, WY 82071, USA \\ Min Huang \\ Villanova University, Villanova, PA 19085, USA
}

\begin{abstract}
.
The AM Herculis binaries (AM Hers) have high and low accretion states. A model is proposed in which the white dwarf's rotation acts like an angular momentum storage battery for the binary orbit. The accreted material from the Roche lobe-overflowing secondary charges up this battery, whereas the magnetic torque of the white dwarf on the secondary discharges it back into the binary's orbit. The Roche lobe's volume becomes alternately larger and smaller than the secondary's volume. This alternation causes the mass flow to vary between a high and low rate.
\end{abstract}

\section{Introduction}

The AM Hers are cataclysmic variables (CVs) in which the white dwarf component has a strong magnetic field. It is generally conjectured that this magnetic field is strong enough to capture the material immediately from the Roche lobeoverflowing secondary and channel it directly onto the white dwarf. Thus, the usual accretion disk in CVs is not formed. Another characteristic of AM Hers is that the orbital period and the white dwarf's rotational period are synchronized or nearly synchronized. The well-observed AM Hers are known to vary in visual luminosity between a high and low state on a time scale of roughly one year. The difference in luminosity is about a factor of 10 . This luminosity is generally attributed to the accretion stream and its impact on the white dwarf. Therefore, the AM Hers seem to vary between a high and low accretion state. See Cropper (1990) for a complete review of AM Hers.

\section{Roche Lobe Overflow}

Under the conditions of mass and angular momentum conservation, if the masslosing star in a binary system is the more massive component, the binary separation will decrease along with the Roche lobe volume of the mass-losing star 
causing a runaway mass exchange. In all well-characterized $\mathrm{CVs}$, the mass-losing star (the secondary) is less massive than the mass-accreting star (the white dwarf primary). If mass and angular momentum are conserved in $\mathrm{CVs}$, then the binary separation and the Roche lobe volume will increase causing the Roche lobe overflow to cease. A number of ideas have been advanced to explain why the secondary continues to overflow its Roche lobe. These include evolutionary expansion, thermal relaxation, or irradiation of the secondary, loss of mass and/or angular momentum from the binary system, magnetic braking, and gravitational radiation. Different mechanisms most likely dominate in different types of CVs or for different binary separations.

Our purpose is not to propose another mechanism for continued overflow but to explore a short term method that stores and later releases orbital angular momentum so that the Roche lobe radius becomes, alternately, smaller and larger than the secondary's radius, causing the overflow to increase and decrease. The appropriate long term mechanism for overflow is still assumed to operate.

\section{The Magnetic Torque Model and Results}

When material from the secondary of an AM Her overflows its Roche lobe, it is immediately captured by the white dwarf's magnetic field. In order for this material to be accreted onto the white dwarf, it must lose most of its angular momentum (approximately the orbital angular momentum of the secondary). This angular momentum is transferred to the white dwarf's rotation via its magnetic field. The smaller the mass ratio of the secondary to the primary is, the more orbital angular momentum is transferred to the white dwarf's rotation. Now, the secondary will be threaded by the white dwarf's magnetic field. When the white dwarf rotates slightly faster than the binary's revolution, its magnetic field torques up the secondary, transferring angular momentum from the white dwarf's rotation back into the orbit. Transferring angular momentum to the orbit causes the binary separation to increase. The Roche lobe radius becomes slightly bigger than the secondary's radius, drastically reducing the mass overflow. The magnetic torque continues to spin down the white dwarf towards synchronization, but there is little mass flow to spin it up. Eventually, the long-term mechanism for overflow causes secondary's radius to again exceed its Roche lobe, and the whole cycle repeats.

An analysis of the torque reveals an oscillatory behavior with a time scale of a few years. The conditions which maximize the opportunity for these torque oscillations are 1) a large magnetic field in the white dwarf, 2) a small binary separation, and 3) a small mass ratio of the secondary to the primary. These conditions match the characteristics of AM Hers. This model results in 1) alternating high/low mass flow rates, 2) essentially synchronous white dwarf rotation and binary revolution, 3) orbital and spin period changes that are too small to be detected, and 4) correlation of the hotspot location with the accretion flow rate.

\section{References}

Cropper, M. 1990, Space Sci. Rev., 54, 195 